Kaare Græsbøll*, Lasse Engbo Christiansen, Uffe Høgsbro Thygesen and

Carsten Kirkeby

\title{
Delaying the peak of the COVID-19 epidemic with travel restrictions
}

https://doi.org/10.1515/em-2020-0042

Received September 29, 2020; accepted February 13, 2021; published online March 10, 2021

\begin{abstract}
:
Objectives: Travel restrictions is an often-used tool for governments to prevent the spread of COVID-19.

Methods: We here used a simple simulation model to investigate the potential effects of travel restrictions within a country.

Results: We found that travel restrictions can delay the peak of the epidemic considerably, but do not affect the spread within the country. We also investigated the effect of implementing travel restrictions early or later in the epidemic, and found that fast implementation is crucial for delaying the epidemic.

Conclusions: Fast implementation of travel restrictions is crucial for delaying the peak of a subsequent outbreak of COVID-19 within a country.
\end{abstract}

Keywords: COVID-19; pandemic; simulation model; travel restrictions; within-country spread.

Travel restrictions are used by countries to control the ongoing COVID-19 pandemic and prevent spread of SarsCoV-2 into countries, and have been successful in countries such as Australia and New Zealand (Costantino, Heslop, and MacIntyre 2020). Limiting the air travel between China and Australia alone has been estimated to avoid 2000 cases and 400 deaths alone (Costantino, Heslop, and MacIntyre 2020). At the same time, travel restrictions are debated worldwide as a violation of international laws (Meier, Habibi, and Yang 2020). Formal recommendations combined with risk assessments and preventive measures to limit the spread of Sars-CoV-2, based on both theoretical end empirical analyses, are needed to reach a sustainable situation for international travel (van Tigerstrom, Halabi, and Wilson 2020). Towards the end of 2020, international travel became more challenged by new lineages of Sars-CoV-2 that spread more rapidly than previous lineages, such as B.1.1.7 (Volz et al. 2020). In the case of Denmark, the numbers of COVID-19 cases rapidly increased during the end of February and beginning of March. Therefore, from March 12 the Danish government rolled out increasingly restrictive control actions to mitigate the disease, such as social distancing, prohibition of major gatherings, cancellation of major public events, quarantine measures and travel restrictions preventing Danish citizens to travel and tourists from other countries to enter Denmark. The goal was to delay the spread of the epidemic over longer time, in order to gain response time and keep the peak case numbers low (Anderson et al. 2020). One of the most discussed control measures for which there is weak evidence from simulation studies, is travel restrictions (Brownstein, Wolfe, and Mandl 2006). One recent simulation study on COVID-19 showed that travel restrictions delayed the outbreak in China by $3-5$ days, but could reduce the spread to other countries with 77\% (Chinazzi et al. 2020). Aleta et al. (2020) used a metapopulation model to show that travel restrictions

*Corresponding author: Kaare Græsbøll, DTU Compute, Technical University of Denmark, 2800, Kongens Lyngby, Denmark, E-mail: kagr@dtu.dk

Lasse Engbo Christiansen and Uffe Høgsbro Thygesen, DTU Compute, Technical University of Denmark, Kongens Lyngby, Denmark

Carsten Kirkeby, Section for Animal Welfare and Disease Control, Department of Veterinary and Animal Sciences, Faculty of Health and Medical Sciences, University of Copenhagen, Frederiksberg, Denmark. https://orcid.org/0000-0001-9292-5526 
between regions in China are effective as short-term prevention but will not help eliminating COVID-19 within a country. Anzai et al. (2020) used a statistical model to study the effect of restricting travelers and from China and found that the implemented lockdown in late January 2020 delayed the epidemic two days in Japan. Lin et al. (2020) used a global network mobility model for Europe and showed that unrestricted travel would have accelerated the spread of COVID-19 in Central Europe. Simulation models always rely on a set of underlying assumptions, and therefore it is important to use different models to investigate the robustness of previous results.

We here used the case of Denmark in the early phase of the epidemic to analyze the practical effects of travel restrictions on the peak size and time of the epidemic.

We used a simple deterministic SEIR simulation model that included domestic transmission within the country and introduction of COVID-19 from Danish travelers returning to DK, to investigate the effects of travel restriction. The model was a differential equation model as described in Keeling and Rohani (2011), parameterized to fit the COVID-19 outbreak in the Danish population with 5.8 mio. people, using an infectious period of seven days; a daily transmission rate of 0.7 (Lin et al. 2020); a latent (exposed) period of 2 days; and we assumed that only $10 \%$ of the cases are detected. The effect of contact restrictions was estimated to $50 \%$ reduction in the transmission rate, based on the model of Chinazzi et al. (2020). We seeded the model with 3020 persons returning to Denmark on March 12th, based on data showing that 341 persons tested positive for COVID-19 and required it from another country (https://files.ssi.dk/COVID19-overvaagningsrapport12032020), assuming only about $10 \%$ of these cases were detected. Based on travel estimations about 20,000 travelers (7,475,429 Danish citizens travelling per year/365 days, data for 2018 from www.dst.dk) were estimated to return to Denmark each day. We assumed that only about $10 \%$ of cases are registered, and thus $10 \%$ of susceptible travelers leaving the country would return infected if no travel ban was implemented in any country. We simulated three scenarios; No intervention; reduced contact between Danish citizens from March 12th; reduced contact and travel ban from March 14th. We also varied the time of implanting the travel ban to seven days earlier and later, to examine the effect of timeliness.

The model results show a clear effect of implementing the travel ban on the peak prevalence (Figure 1). Without intervention, the simulated peak epidemic would arrive on April 17th and result in maximal 100,000 infected at the same time. Implementing reduced contact between citizens decreased the peak size to under 50,000 infected at the same time, and delayed the peak of the epidemic to May 1st (14 days delay). Implementing a strategy with reduced contact restrictions and travel restrictions resulted in a delay of the peak epidemic to May 14th (27 days delay). Hesitation with implementation of travel restrictions for 7 days resulted

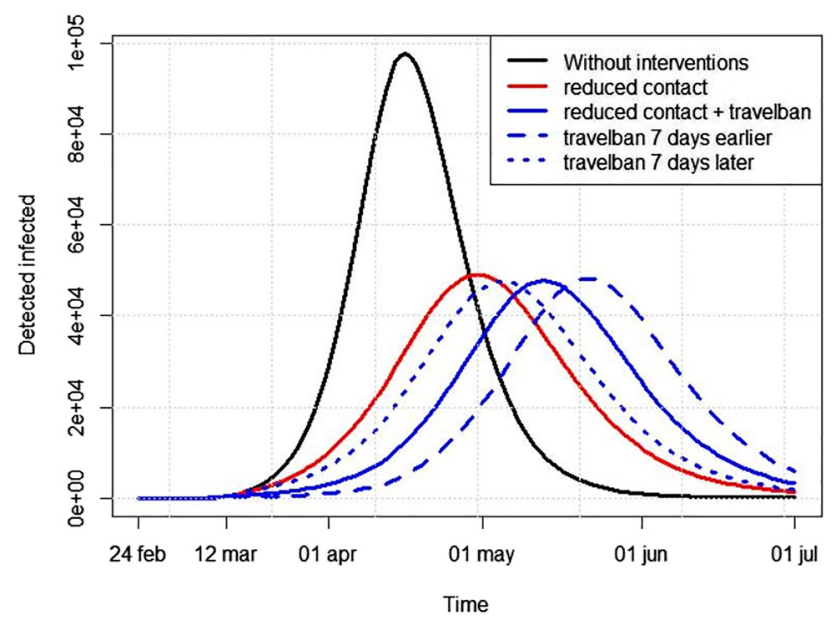

Figure 1: The number of detected cases over time in the different modeled scenarios. The black line shows the epidemic without interventions. The red line shows the reduced contact control action implemented on March 12. The blue line shows the combination of reduced contact and travel ban implemented from March 14. The blue dotted line and dashed line shows the same, but with travel ban implemented on March 7 and March 21, respectively. 
in a peak epidemic on May 6th (8 days earlier). Implementing the travel restrictions 7 days before delayed the peak epidemic until May 23rd (9 days delay).

Given the assumptions made, these results support the findings of Aleta et al. (2020), Adekunle et al. (2020) and Lin et al. (2020), as travel restrictions are found effective for delaying the epidemic, but will not affect the peak size. Similarly, Clifford et al. (2020) found that control measures combining exit and entry screening are able to delay the epidemic 7-8 days (mean). An underlying assumption for this delay is that the transmission from incoming travelers is larger than the transmission within the country. Furthermore, as shown by Clifford et al. (2020), the delay is inversely proportional to the number of infected persons travelling into a country. This highlights the effect of preventive measures when a country opens up for international travel, which is up to a $92.1 \%$ reduction in secondary cases if 14-day isolation period of positive cases is implemented (Dickens et al. 2020).

The introduction of travel restrictions is costly for a society. Thus it is important to balance between delaying the epidemic enough to gain response time, and not delaying the epidemic too long because the costs for the control actions will be prolonged. Both extreme cases will be suboptimal. Furthermore, the optimum onset of travel restrictions is affected by many other factors such as the fraction of the population that has less susceptibility; the duration of immune status (recovered) after infection; risk of (re-)introduction from other countries; and the effectivity of preventive measures such as exit and entry screening (Clifford et al. 2020; Dickens et al. 2020).

Travel restrictions between two countries will only have an impact when prevalence differs between these countries. If two adjacent countries are able to keep transmission low, they can form a "travel bubble", allowing free travel between them (van Tigerstrom, Halabi, and Wilson 2020). This can delay the epidemic, for instance until sufficient vaccination has been implemented. If the prevalence differs considerably between any two countries, limiting travel between them is an effective way to keep incoming cases to a minimum and delay the spread of incoming transmission. This study highlight the importance of travel restrictions so that specific restrictions and screenings aimed at reducing export from countries with high prevalence could still be effective. This theoretical study confirms that early travel restrictions is a rational choice for a government wishing to delay the epidemic in its country, and thus supports the decision of governments implementing travel restrictions.

Acknowledgements: The authors would like to thank Danmarks Statistik (www.dst.dk) for making travel figures available.

Research funding: None declared.

Author contribution: All authors have accepted responsibility for the entire content of this manuscript and approved its submission.

Competing interests: Authors state no conflict of interest.

Informed consent: Informed consent was obtained from all individuals included in this study.

Ethical approval: No ethical approvals are needed for this study.

\section{References}

Adekunle, A., M. Meehan, D. Rojas-Alvarez, J. Trauer, and E. McBryde. 2020. "Delaying the COVID-19 Epidemic in Australia: Evaluating the Effectiveness of International Travel Bans.” Australian and New Zealand Journal of Public Health 44 (4): 257-9.

Aleta, A., Q. Hu, J. Ye, P. Ji, and Y. Moreno. 2020. “A Data-Driven Assessment of Early Travel Restrictions Related to the Spreading of the Novel COVID-19 within Mainland China.” Chaos, Solitons \& Fractals 139: 110068.

Anderson, R. M., H. Heesterbeek, D. Klinkenberg, and T. D. Hollingsworth. 2020. "How Will Country-Based Mitigation Measures Influence the Course of the COVID-19 Epidemic?” The Lancet 395 (10228): 931-4.

Anzai, A., T. Kobayashi, N. M. Linton, R. Kinoshita, K. Hayashi, A. Suzuki, and H. Nishiura. 2020. "Assessing the Impact of Reduced Travel on Exportation Dynamics of Novel Coronavirus Infection (COVID-19).” Journal of Clinical Medicine 9 (2): 601.

Brownstein, J. S., C. J. Wolfe, and K. D. Mandl. 2006. "Empirical Evidence for the Effect of Airline Travel on Inter-regional Influenza Spread in the United States.” PLoS Medicine 3 (10): e401. 
Chinazzi, M., J. T. Davis, M. Ajelli, C. Gioannini, M. Litvinova, S. Merler, A. P. Piontti, K. Mu, L Rossi, C. Viboud, X. Xiong, H. Yu, M. E. Halloran, I. M. Longini, Jr., and A. Vespignani. 2020. "The Effect of Travel Restrictions on the Spread of the 2019 Novel Coronavirus (COVID-19) Outbreak.” Science 368: 395-400.

Clifford, S., C. A. Pearson, P. Klepac, K. Van Zandvoort, B. J. Quilty, R. M. Eggo, and S. Flasche, CMMID COVID-19 Working Group. 2020. “Effectiveness of Interventions Targeting Air Travellers for Delaying Local Outbreaks of SARS-CoV-2." Journal of Travel Medicine 27 (5): taaa068.

Costantino, V., D. J. Heslop, and C. R. MacIntyre. 2020. "The Effectiveness of Full and Partial Travel Bans against COVID-19 Spread in Australia for Travellers from China during and after the Epidemic Peak in China.” Journal of Travel Medicine 27 (5): taaa081.

Dickens, B. L., J. R. Koo, J. T. Lim, H. Sun, H. E. Clapham, A. Wilder-Smith, and A. R. Cook. 2020. "Strategies at Points of Entry to Reduce Importation Risk of COVID-19 Cases and Reopen Travel.” Journal of Travel Medicine 27 (8): taaa141.

Keeling, M. J., and P. Rohani. 2011. Modeling Infectious Diseases in Humans and Animals. New Jersey: Princeton University Press. Lin, Q., S. Zhao, D. Gao, Y. Lou, S. Yang, S. Musa, M.H. Wang, Y. Cai, W. Wang, L. Yang, and D. He. 2020. “A Conceptual Model for the Coronavirus Disease 2019 (COVID-19) Outbreak in Wuhan, China with Individual Reaction and Governmental Action.” International Journal of Infectious Diseases 93: 211-216.

Meier, B. M., R. Habibi, and Y. T. Yang. 2020. “Travel Restrictions Violate International Law.” Science 367 (6485): 1436.

van Tigerstrom, B. J., S. F. Halabi, and K. R. Wilson. 2020. “The International Health Regulations (2005) and the Re-establishment of International Travel amidst the COVID-19 Pandemic.” Journal of Travel Medicine 27 (8): taaa127.

Volz, E., S. Mishra, M. Chand, J. C. Barrett, R. Johnson, L. Geidelberg, W. R. Hinsley, D. J. Laydon, G. Dabrera, A. O’Toole, R. Amato, M. Ragonnet-Cronin, I. Harrison, B. Jackson, C. V. Ariani, O. Boyd, N. Loman, J. T. McCrone, S. Gonçalves, D. Jorgensen, R. Myers, V. Hill, D. K. Jackson, K. Gaythorpe, N. Groves, J. Sillitoe, D. P. Kwiatkowski, S. Flaxman, O. Ratmann, S. Bhatt, S. Hopkins, A. Gandy, A. Rambaut, N. M. Ferguson, and COG-UK. 2021. "Transmission of SARS-CoV-2 Lineage B.1.1. 7 in England: Insights from Linking Epidemiological and Genetic Data.” medRxiv, 2020-12. 